\title{
Population pharmacokinetics of fluconazole given for secondary prevention of oropharyngeal candidiasis in HIV-positive patients
}

Received: 5 June 2001 / Accepted in revised form: 23 August 2001 / Published online: 30 October 2001

(C) Springer-Verlag 2001

\begin{abstract}
Objectives: To determine fluconazole population pharmacokinetics and explore the relationships between fluconazole average concentration and treatment effectiveness or microbiological resistance induction during a study aimed at evaluating the efficacy, tolerability and resistance induction after secondary prevention with fluconazole $(150 \mathrm{mg}$ weekly) versus placebo in human immunodeficiency virus-positive $(\mathrm{HIV}+)$ patients with oropharyngeal candidiasis.

Methods: Population pharmacokinetic parameters of fluconazole determined from 458 serum drug concentration measurements obtained over 37 months in 132 $\mathrm{HIV}+$ patients not receiving highly active antiretroviral therapy. Mean estimates and variabilities were generated using non-linear regression analysis. Logistic and linear regression analyses were used to explore the relationships between the estimated average concentration of fluconazole and candidiasis relapse or fungal resistance towards fluconazole.

Results: Fluconazole kinetics were best described by a one-compartment model with first-order oral absorp tion from the gastrointestinal tract. The pharmacokinetics were influenced only by body weight. No effect was observed for gender, age, height or lymphocyte CD4 counts. The mean apparent population clearance was $0.79 \mathrm{l} / \mathrm{h}$, the volume of distribution 571 and the absorption constant $\left(\mathrm{k}_{\mathrm{a}}\right) 0.93 \mathrm{~h}^{-1}$. Inter-occasion variability in clearance $(45 \%)$ was large relative to inter-
\end{abstract}

C. Csajka $(\bowtie) \cdot$ L.A. Décosterd · T. Buclin · J. Biollaz Division of Clinical Pharmacology,

University Hospital, CHUV, Lausanne, Switzerland

E-mail: chantal.csajka@chuv.hospvd.ch

Tel.: + 41-21-3144257

Fax: +41-21-3144266

J.-L. Pagani · J. Bille

Division of Infectious Diseases,

University Hospital CHUV, Lausanne, Switzerland

K. Fattinger

Division of Clinical Pharmacology and Toxicology,

University Hospital, Zurich, Switzerland subject variability $(21 \%)$. Taking into account the average fluconazole concentration or the time above the minimal inhibitory concentrations did not clinically improve the prediction of the occurrence of oropharyngeal relapse or microbiological resistance.

Conclusion: The relationship between fluconazole concentrations and preventive effectiveness was poor. Together with the rather large inter-occasion variability in fluconazole clearance, this suggests no role of therapeutic drug monitoring in optimising fluconazole treatment for secondary prevention.

Keywords Fluconazole $\cdot$ PK $\cdot$ Population · Candidiasis $\cdot$ HIV $\cdot$ Patients

\section{Introduction}

Oropharyngeal candidiasis is observed in many human immunodeficiency virus-positive $(\mathrm{HIV}+)$ patients and still represents the most frequent opportunistic infection in HIV-infected individuals. The efficacy and safety of fluconazole in the treatment of oral thrush associated with HIV infection has been demonstrated [1, 2], but the frequency of relapses is high and increases with the patient immunodeficiency. The management of oropharyngeal candidiasis in HIV + patients using a secondary prevention with fluconazole $(50-150 \mathrm{mg}$ once a week) has thus been proposed, and the efficacy and safety of this approach are now established $[3,4,5]$. This strategy presents advantages with respect to cost, compliance and drug interactions [6]. However, increased resistance to fluconazole has been reported during prophylactic therapy [7].

A prospective, double-blind, randomised, placebomatched study was conducted in a cohort of HIV + patients over a period of 37 months to assess the effect of secondary prevention by weekly fluconazole on the development of clinical and microbiological resistance to fluconazole (Pagani et al., unpublished observations). Blood samples were collected during this study primarily 
to assess patient's adherence to treatment. The aims of this analysis were to characterise the population pharmacokinetics of fluconazole in this group of patients. As a secondary exploratory endpoint, we evaluated the relationships between the average blood concentrations or the time over the minimal inhibitory concentrations (MICs; according to a posteriori Bayesian estimation) and the probability of oropharyngeal candidiasis relapse or of clinical or microbiological resistance to fluconazole.

\section{Materials and methods}

Patients

Data from 132 patients with 458 serum concentrations were available for the population pharmacokinetic analysis. The patients were randomised to receive orally either fluconazole $(150 \mathrm{mg}$ weekly; $n=66)$ or placebo $(n=66)$. In case of candidiasis relapse, a treatment of fluconazole ( $200 \mathrm{mg}$ per day) was undertaken for 7 days. Serum samples were drawn at least every 3 months on follow-up visits and during candidiasis relapses to check for compliance. For convenience, blood samples were taken at each visit. No predefined sampling strategy was applied, but the exact time of the last administered dose was recorded by the physician at each visit to get accurate dosing information. In addition to accurate dosing information and time of sampling, the following data were collected for each patient: gender, body weight (BW), height, age, number of CD4 lymphocytes and serum creatinine concentration. There were 93 males and 39 females. Their body weights ranged from $41 \mathrm{~kg}$ to $97 \mathrm{~kg}$ (mean $61 \mathrm{~kg}$ ), their height from $146 \mathrm{~cm}$ to $192 \mathrm{~cm}$ (mean $172 \mathrm{~cm}$ ) and their age from 25 years to 63 years (mean 37 years). The serum creatinine concentration ranged from $53 \mu \mathrm{mol} / \mathrm{l}$ to $181 \mu \mathrm{mol} / 1$ (mean $83 \mu \mathrm{mol} / \mathrm{l}$ ); the mean creatinine clearance estimated using the equation of Cockcroft and Gault [8] was $85 \mathrm{ml} / \mathrm{min}$ (five patients $<60 \mathrm{ml} / \mathrm{min}$ ). The CD4 lymphocytes ranged from $0 / \mathrm{mm}^{3}$ to $605 / \mathrm{mm}^{3}$ (mean $101 / \mathrm{mm}^{3}$ ). The number of fluconazole serum concentration measurements per patient was 2.7 (range 1-8).

\section{Analytical methods}

Serum fluconazole concentrations were measured using reversephase high-performance liquid chromatography after solid-phase extraction, adapted from the method published by Inagaki [9]. The chromatographic equipment consisted of a Hewlett-Packard 1090 instrument (Series II; Hewlett-Packard, Germany) equipped with a spectrophotometric ultraviolet-visible (UV-VIS) diode-array detector (DAD) set at $200 \mathrm{~nm}$. The separations were carried out on a Macherey-Nagel ChromCart 125/4 Nucleosil 100 C18AB (Düren, Germany) using an isocratic elution of acetonitrile $+0.1 \%$ acetic acid/bidistilled water (17/83) for $10 \mathrm{~min}$ at a flow rate of $1 \mathrm{ml} / \mathrm{min}$, followed by rinsing and equilibration steps. The calibrations, using the internal standard method (UK-54373) are linear $\left(\mathrm{r}^{2}>0.999\right)$ over the $0.1-$ to $25-\mathrm{mg} / \mathrm{l}$ concentration range. The detection limit was $0.1 \mathrm{mg} / \mathrm{l}$. Quality control samples at $0.75-$, 7- and $20-\mathrm{mg} / 1$ concentrations had, overall, an inter-day relative standard deviation within $1.4-7.5 \%$. The mean inter-assay deviations from their nominal concentrations were comprised within the range $-3.3 \%$ to $+0.4 \%$. Calculated precision and accuracy of the analytical method were therefore in accordance with the $\pm 15 \%$ recommendations of the Conference Report on Bioanalytical Method Validation [10].

\section{Population pharmacokinetic analysis}

The analysis was performed using the computer program NONMEM, version V, developed by Beal and Sheiner [11]. It uses mixed-effects (fixed and random) non-linear regression modelling to estimate the mean and the variance of the pharmacokinetic parameters in the study population and factors that may influence them. A stepwise procedure was used to find the model that best fitted the data. First, we compared one- and two-compartment models with first- or zero-order absorption. The influence of each recorded patient characteristics on the kinetic parameters was tested sequentially. A proportional error distribution was assumed for the inter-individual variability of the pharmacokinetic parameters of the form shown below:

$C L_{j}=T V C L \cdot\left(1+\eta_{j}^{C L}\right)$

$V d_{j}=T V V \cdot\left(1+\eta_{j}^{V d}\right)$

$K a_{j}=T V K a \cdot\left(1+\eta_{j}^{K a}\right)$

in which $\mathrm{CL}_{\mathrm{j}}, \mathrm{Vd}_{\mathrm{j}}$ and $\mathrm{Ka}_{\mathrm{j}}$ are the true values of the pharmacokinetic parameters for the $\mathrm{j}^{\text {th }}$ individual; TVCL, TVVd and TVKa are the typical values of the pharmacokinetic parameters in the population and $\eta$ is a random effect with mean zero and variance $\omega^{2}$. No covariance was assumed between the parameters.

To allow for an evaluation of pharmacokinetic-pharmacodynamic relationships, an additional inter-occasion variability $\left(\eta_{2 i j}^{P} \cdot O c c_{1}+\eta_{3 i j}^{P} \cdot O c c_{2}+\ldots+\eta_{n i j}^{P} \cdot O c c_{n}\right)$ was introduced on clearance, although only one concentration sample per occasion was available, thus making inter-occasion and intra-individual variabilities confounded. The equation of the inter-individual and interoccasion variability of the $\mathrm{j}^{\text {th }}$ subject was expressed as follows [12]:

$C L_{j}=T V C L \cdot\left(1+\eta_{1 i j}^{C L}+\eta_{2 i j}^{C L} \cdot O c c_{1}+\eta_{3 i j}^{C L} \cdot O c c_{2}+\ldots+\eta_{n i j}^{C L} \cdot O c c_{n}\right)$

The parameter $\operatorname{Occ}_{n}$ takes the value of 1 on the $n^{\text {th }}$ occasion and 0 otherwise, and the occasion-related variations, $\eta_{2 \mathrm{ij}}$ to $\eta_{\mathrm{nij}}$, are constrained to have the same variance. An additive and proportional error distribution was assumed for description of the intraindividual (residual) variability: $Y_{j}=F_{j}+F_{j} \cdot \varepsilon_{1 j}+\varepsilon_{2 j}$ where $\mathrm{F}_{\mathrm{j}}$ is the model prediction concentration for the $\mathrm{j}^{\text {th }}$ individual, $\epsilon_{1}$ and $\epsilon_{2}$ are the residual intra-individual error terms with mean zero and variance $\sigma^{2}$. All drug concentration data below the detection limit were set to half the detection limit. To limit their influence, the variance of the additive intra-individual error component was forced to equal or exceed the squared half of the detection limit. the difference in the minimum value of the objective function $(\Delta \mathrm{OF})$ was used to compare two models.

For model selection, we compared the plots of predicted response versus observations and used the Akaike criterion [13]. In addition, the following goodness-of-fit parameters were considered when choosing between models: residual plots, standard error and correlation matrix of the parameter estimates, size of the interindividual variance of the pharmacokinetic parameters, and size of the residual error. A simulation based on the pharmacokinetic results including 1000 individuals was performed using NONMEM to calculate the $95 \%$ predicted interval depicted in Fig. 1 . The concentrations at the 2.5 th and 97.5 th percentile at each time point were retrieved to construct the prediction interval.

\section{Analysis of effect data}

Oropharyngeal candidiasis relapse was determined clinically and confirmed by means of bacteriological examinations (direct examination, primary strain isolation, identification of colonies). The susceptibility of Candida Albicans towards fluconazole was determined at each visit with a disk diffusion agar test using $50 \mu \mathrm{g}$ fluconazole [7]. The inhibition diameter $(\mathrm{mm})$ was measured, and microbiological resistance was defined as an inhibition diameter on an agar plate smaller than $25 \mathrm{~mm}$.

The individual average concentrations of fluconazole were estimated using the post-hoc estimates of clearance. Both the area 


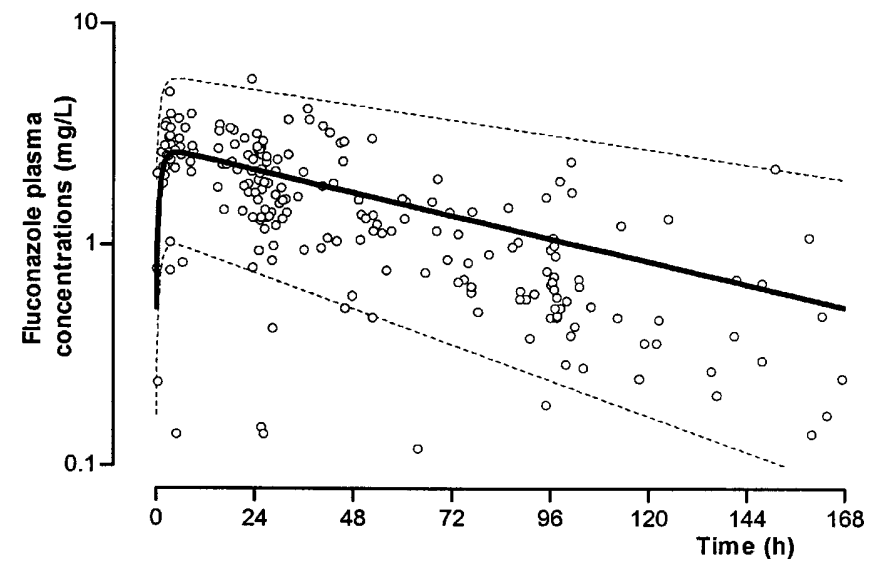

Fig. 1 Fluconazole plasma concentrations in 66 human immunodeficiency virus-positive patients receiving $150 \mathrm{mg}$ fluconazole per week (open circles), with the average population prediction (solid line) and $95 \%$ prediction interval (dashes lines)

under the concentration-time curve (AUC) and the time over the MIC (defined as $0.39 \mathrm{mg} / 1$ for C. albicans) were used for the statistical analysis. The AUC was defined as $D_{\text {ose }} / C L$ and the time above MIC was simulated using the post-hoc individual pharmacokinetic estimates in the software program Excel (version 7, Windows 98). The relationship between fluconazole AUC or time above MIC and candidiasis relapses was assessed using logistic regression. To take into account a potential difference in the time until relapse between both groups, a weighting variable was also introduced in the analysis. The independent variables were treatment (fluconazole or placebo) and fluconazole AUC or time above MIC, the dependent variable the candidiasis relapse and the weighting variable the logarithm of the time until relapse. The resistance to treatment was assessed using linear regression, with the inhibition diameter $(\mathrm{mm})$ defined as the dependent variable and treatment (nested within subject) and fluconazole AUC or time above MIC as the independent variables. All the standard statistical tests were performed using the Statistix software (version 4.1).

\section{Results}

Population pharmacokinetic analysis

A one-compartment model with first-order absorption from the gastrointestinal tract was found to describe the data adequately (two- vs one-compartment model: $\left.\Delta \mathrm{OF}=0.0, \Delta \mathrm{n}_{\mathrm{par}}=2\right)$. The absorption kinetics were difficult to estimate with precision since only trough concentration samples were generally taken. An intersubject variability was assigned on apparent clearance (CL) $\left(\Delta \mathrm{OF}=-17.0, \Delta \mathrm{n}_{\mathrm{par}}=1\right)$ and on apparent volume of distribution $\left(\mathrm{V}_{\mathrm{d}}\right)\left(\Delta \mathrm{OF}=-7.9, \Delta \mathrm{n}_{\mathrm{par}}=1\right)$. Without any covariates, the population estimate of $\mathrm{CL}$ was $0.65 \mathrm{l} / \mathrm{h}$. The inter-subject variability in this parameter, expressed as percentage coefficient of variation $(\mathrm{CV} \%)$, was $34 \%$; when allowing for an inter-subject and inter-occasion variability, the $\mathrm{CV}$ values were $30 \%$ and $45 \%$, respectively $(\Delta \mathrm{OF}=-186.0)$. The mean population $\mathrm{V}_{\mathrm{d}}(\mathrm{CV} \%)$ was $70.71(30 \%)$. The absorption was rapid, with a halflife of $42 \mathrm{~min}$. The additive and proportional intra- subject variability were $0.78 \mathrm{mg} / 1(\mathrm{SD})$ and $34 \%(\mathrm{CV} \%)$, respectively.

\section{Covariates assessment}

The relationship between various covariates and the individual estimates of fluconazole $\mathrm{CL}$ and $\mathrm{V}_{\mathrm{d}}$ were examined. Body weight significantly influenced both the $\mathrm{CL}$ and the $\mathrm{V}_{\mathrm{d}}$ of fluconazole $\left(\Delta \mathrm{OF}>-14.4, \Delta \mathrm{n}_{\mathrm{par}}=1\right)$, reducing the $\mathrm{CL}$ variability from $34 \%$ to $31 \%$ and explaining the overall variability on the $\mathrm{V}_{\mathrm{d}}$. The regression model for fluconazole $C L$ and $\mathrm{V}_{\mathrm{d}}$ accounting for BW was $C L=0.79+1.1 \times B W E$ and $V_{d}=87.4+44.2 \times B W E$, where $B W E$ expresses the relative deviation of the individual $\mathrm{BW}$ from the mean $\mathrm{BW}$ in the population $(B W E=-1+B W /$ mean $B W)$. No effect of age $(\triangle \mathrm{OF}=$ $\left.-0.3, \Delta \mathrm{n}_{\mathrm{par}}=1\right)$ or gender $\left(\Delta \mathrm{OF}=0.0, \Delta \mathrm{n}_{\mathrm{par}}=1\right)$ was observed. The number of lymphocytes CD4 and the height influenced the $\mathrm{CL}$ of fluconazole $(\Delta \mathrm{OF}>-3.3$, $\left.\Delta \mathrm{n}_{\mathrm{par}}=1\right)$ but did not remain statistically significant in the multivariate analysis $\left(\Delta \mathrm{OF}=-1, \Delta \mathrm{n}_{\mathrm{par}}=1\right)$, being too highly correlated with body weight. Similarly, no effect of creatinine $\mathrm{CL}$ on fluconazole $\mathrm{CL}$ was observed $\left(\Delta \mathrm{OF}=-0.3, \Delta \mathrm{n}_{\mathrm{par}}=1\right)$. The remaining inter-subject and inter-occasion variabilities in CL were, respectively, $21 \%$ and $45 \%$. The values of the population parameters for the final regression model are given in Table 1. Plasma concentrations of fluconazole with population prediction and 95\% prediction interval are presented in Fig. 1.

\section{Fluconazole effects}

The population pharmacokinetic analysis was used to assess whether taking into account either the average concentration of fluconazole or the time above MIC could improve the prediction of the response to fluconazole prophylactic treatment. As assessed by Pagani et al. (unpublished observations), significantly more patients experienced a relapse in the placebo than in the fluconazole group ( $90 \%$ vs $61 \%, P=0.001)$. The average fluconazole concentration failed to improve the prediction of the response $(P=0.7)$, even while using the time interval to relapse as a weighting variable $(P=0.5)$. Similarly, the time above MIC failed to predict the response $(P=0.8)$. A tendency to a significant difference could be detected between both groups for the occurrence of microbiological resistance in Candida isolates $(P=0.03)$. However, when considering the millimetres of inhibition measured in the disk diffusion agar test, a difference of $3.2 \mathrm{~mm}$ diameter was noticed between the placebo (mean $46.5 \mathrm{~mm}$ ) and the fluconazole $(43.3 \mathrm{~mm}$, $P<0.0001)$ groups. This trend towards some decrease in susceptibility to fluconazole is however small, far from the cut-off value of $25 \mathrm{~mm}$ defined for microbiological resistance to treatment and, therefore, probably not clinically significant. Here, again, taking into account the average fluconazole concentration or the time above 
Table 1 Population pharmacokinetic parameters of oral fluconazole. Mean population estimates. All parameters are apparent values. $C L$ apparent clearance, $V_{d}$ apparent volume of distribution, $k_{a}$ absorption constant

\begin{tabular}{|c|c|c|c|c|c|c|}
\hline \multirow[t]{2}{*}{ Parameter } & \multicolumn{2}{|c|}{ Population mean } & \multicolumn{2}{|c|}{$\begin{array}{l}\text { Inter-individual } \\
\text { variability }^{\mathrm{a}}\end{array}$} & \multicolumn{2}{|c|}{$\begin{array}{l}\text { Inter-occasion } \\
\text { variability }^{\mathrm{a}}\end{array}$} \\
\hline & Estimate & SEM $^{\mathrm{b}}$ & Estimate & SEM $^{\mathrm{b}}$ & Estimate & SEM $^{\mathrm{b}}$ \\
\hline CL (l/h) & 0.79 & $4 \%$ & $21 \%$ & $49 \%$ & $45 \%$ & $53 \%$ \\
\hline $\mathrm{CL}_{\mathrm{bw}}^{\mathrm{d}}(\mathrm{l} / \mathrm{h})$ & 1.14 & $17 \%$ & & & & \\
\hline $\mathrm{Vd}(1)$ & 57.4 & $6 \%$ & & & & \\
\hline $\mathrm{Vd}_{\mathrm{bw}}{ }^{\mathrm{d}}(\mathrm{l} / \mathrm{h})$ & 44.2 & $29 \%$ & & & & \\
\hline $\mathrm{Ka}\left(\mathrm{h}^{-1}\right)$ & 0.93 & $19 \%$ & & & & \\
\hline$\sigma_{\text {add }}\left(\mathrm{mg} / \mathrm{l}^{\mathrm{e}}\right.$ & 0.19 & $17 \%$ & & & & \\
\hline$\sigma_{\text {prop }}(\mathrm{CV} \%)^{\mathrm{e}}$ & $31 \%$ & $45 \%^{\mathrm{c}}$ & & & & \\
\hline
\end{tabular}

${ }^{\mathrm{a}}$ Estimates of variability expressed as coefficient of variation

${ }^{\mathrm{b}} \mathrm{S}$ tandard error of the estimates, expressed as coefficient of variation

${ }^{\mathrm{c}}$ Standard error of the variance components, taken as $\sqrt{\text { s.e }_{\text {estimate }} / \text { estimate, }}$, expressed as a percentage ${ }^{\mathrm{d}}$ Proportionality term relating CL and Vd to a relative increase or decrease in body weight $(\mathrm{kg})$ from the average value $(61 \mathrm{~kg})$ in the population

${ }^{\mathrm{e}}$ Residual intra-individual variability of the serum concentration, expressed as standard deviation (add, $\mathrm{SD} \mathrm{mg} / \mathrm{l}$ ) and coefficient of variation (prop, $\mathrm{CV} \%$ )
MIC did not improve statistically the prediction of this effect.

\section{Discussion and conclusions}

This study determined the population pharmacokinetics of fluconazole and demonstrated that only BW but not gender nor any of the other studied covariates influences fluconazole pharmacokinetics. Intra-individual as opposed to inter-subject variability in CL was rather large, and individual fluconazole concentrations did not correlate with therapeutic outcome, indicating that therapeutic drug monitoring would probably not improve use effectiveness of fluconazole.

The mean population pharmacokinetic parameter estimates were in the same range as those already reported $[14,15,16,17]$. Although a previous study reported no effect of BW on fluconazole pharmacokinetics [16], in this study BW significantly influenced fluconazole $C L$ and $V_{d}$. In contrast to previous studies $[16,18]$, this study included female and male patients. Since neither the covariate plots suggested any gender differences nor the inclusion of additional parameters for gender differences in CL and/or $\mathrm{V}_{\mathrm{d}}$ improved the fit, a clinically relevant effect of gender on fluconazole pharmacokinetics can be excluded.

A reduction in fluconazole dose was previously suggested for people with HIV infection who are seriously ill and/or who have compromised renal function $[16,18]$. However, in this study, after inclusion of BW, neither lymphocyte CD4 count nor creatinine CL exhibited a significant effect on fluconazole CL. The initially detected statistical significance of CD4 counts in our analysis probably occurred due to an inverse correlation of lymphocyte CD4 counts - as a marker surrogate of disease progression - and BW. A possible explanation why this study failed to detect a relevant effect of creatinine $\mathrm{CL}$ on fluconazole $\mathrm{CL}$, despite the fact that fluconacole is mainly excreted unchanged renally, might be that this study included only a few patients with moderately impaired and nobody with severely impaired renal function. Therefore, a dose reduction in patients with considerably impaired renal function still seems justified, although we would not recommend the same for patients with low CD4 counts.

In contrast to a previous population pharmacokinetic study on fluconazole, which mainly included only one sampling occasion per patient, this study included blood sampling on several occasions for most patients. This sampling schedule allowed us to distinguish between inter-subject and inter-occasion variability. After inclusion of the covariate $\mathrm{BW}$, the remaining intersubject variability was rather small compared with the inter-occasion variability. No relationship between the patients average fluconazole concentration and the occurrence of oropharyngeal relapse or clinical or microbiological resistance could be detected. The large inter-occasion variability of pharmacokinetics might explain why drug concentrations at one occasion failed to explain overall fluconazole response. However, also, the sparse sample collection may have compromised the power of the study to detect association of limited strength. In conclusion, this study demonstrated that fluconazole pharmacokinetics are gender independent and do not depend on CD4 counts. The rather large inter-occasion variability in fluconazole $\mathrm{CL}$ and the absent correlation between drug exposure and treatment response suggests that therapeutic drug monitoring does not represent a sensible approach for improving fluconazole's therapeutic effectiveness.

Acknowledgements The analytical part of this study was supported by Pfizer AG (Switzerland). Karin Fattinger is supported by a SCORE A Career Development Award (no. 3200-052190.97) from the Swiss National Science Foundation. We are indebted to Natacha Bélaz for excellent technical assistance in the laboratory.

\section{References}

1. Martin MV (2000) The use of fluconazole and itraconazole in the treatment of Candida albicans infections: a review. J Antimicrob Chemother 44:429-437 
2. De Wit S (1989) Comparison of fluconazole and ketoconazole for oropharyngeal candidiasis in AIDS. Lancet 1:746-747

3. Marriott DJE, Jones PD, Hoy JF, Speed BR, Harkness JL (1993) Fluconazole once a week as secondary prophylaxis against oropharyngeal candidiasis in HIV-infected patients. A double-blind placebo-controlled study. Med J Aust 158:312-315

4. Esposito R, Uberti F (1990) Maintenance therapy of oropharyngeal candidiasis in HIV infected patients with fluconazole. AIDS 4:1033-1035

5. Leen CL, Dunbar EM, Ellis ME, Mandal BK (1990) Onceweekly fluconazole to prevent recurrences of oropharyngeal candidiasis in patients with AIDS or AIDS-related-complex: a double-blind study. J Infect 21:55-60

6. Chave JP, Bille J, Glauser M (1989) Single-dose therapy for oral candidiasis with fluconazole in HIV-infected adults: a pilot study. J Infect Dis 159:806-807

7. Troillet N, Durussel C, Bille J, Glauser M, Chave JP (1993) Correlation between in vitro susceptibility of Candida albicans and fluconazole resistant oropharyngeal candidiasis in HIVinfected patients. Eur J Clin Microbiol Infect Dis 12:911-915

8. Cockcroft D, Gault MH (1976) Prediction of creatinine clearance from serum creatinine. Nephron 16:31-41

9. Inagaki K, Takagi J, Okamoto MP, Gill MA (1992) Determination of fluconazole in human serum by solid-phase extraction and reverse-phase high-performance liquid chromatography. Ther Drug Monit 14:306-311
10. Karnes TH, Shiu G, Shah VP (1991) Validation of bioanalytical methods. Pharm Res 8:421-426

11. Beal S, Sheiner L (1980) The NONMEM system. Am Stat 34:118-119

12. Karlsson MO, Sheiner LB (1993) The importance of modelling interoccasion variability in population pharmacokinetic analyses. J Pharmacokinet Biopharm 21:735-750

13. Akaike H (1974) A new look at the statistical model identification. IEEE Trans Automat Contr 19:716-723

14. Debruyne D, Ryckelynck JP (1993) Clinical pharmacokinetics of fluconazole. Clin Pharmacokinet 24:10-27

15. DeMuria D, Forrest A, Rich J, Scavone JM, Cohen LG, Kazanjian PH (1993) Pharmacokinetics and bioavailability of fluconazole in patients with AIDS. Antimicrob Agents Chemother 37:2187-2192

16. McLachlan AJ, Tett SE (1996) Pharmacokinetics of fluconazole in people with HIV infection: a population analysis. Br J Clin Pharmacol 41:291-298

17. Debruyne D (1997) Clinical pharmacokinetics of fluconazole in superficial and systemic mycoses. Clin Pharmacokinet 33:52-77

18. Tett S, Moore S, Ray J (1995) Pharmacokinetics and bioavailability of fluconazole in two groups of males with human immunodeficiency virus (HIV) infection compared with those in a group of males without HIV infection. Antimicrob Agents Chemother 39:1835-1841 